\title{
SCLEROTHERAPY CONTRAINDICATIONS, SEQUELAES, AND COMPLICATIONS - INFORMING PATIENTS PROPERLY AND OBJECTIVELY IS REQUIRED
}

\author{
Tomasz Urbanek', Maciej Piotr Rzepka², Agnieszka Jolanta Roj², \\ Patrycja Alicja Rozwadowska² \\ 'Department of General Surgery, Vascular Surgery, Angiology, and Phlebology, \\ Medical University of Silesia, Katowice, Poland \\ ${ }^{2}$ Student's Scientific Association of Department of General Surgery, Vascular Surgery, \\ Angiology, and Phlebology, Medical University of Silesia, Katowice, Poland
}

\begin{abstract}
Introduction: The objective of the article is the analysis of the information provided to patients by medical centres that offer sclerotherapy in Poland, as well as its possible impact on patients' decisions about the procedure and the choice of a clinic.

Material and methods: An analysis of the data obtained from web page information available from 212 medical centres in Poland proposing sclerotherapy treatment. The medical centres were divided into 5 categories: aesthetic clinics, medical centres offering varicose vein treatment, medical and aesthetic centres, private specialist practices, and hospitals. The data provided by medical centres were divided into 5 main categories: main information about the procedure, indication description, information about the contraindications, post-procedure care, and possible complications.

Results: In the analysed material, only $34 \%$ of medical centres informed potential patients about contraindications and only $37 \%$ about possible procedure complications, in their web pages. The most commonly presented complications were haematoma and temporary discoloration of skin, and the possibility of venous thromboembolism occurrence was mentioned only by $10 \%$. Information about the treatment not always being effective was provided by $16 \%$ of websites, and only $42.5 \%$ informed patients about the possible need of repeated procedures.

Conclusions: The results show that in many centres sclerotherapy is considered to be an aesthetic rather than medical procedure. To attract patients, several medical centres inform them only about the indications rather than contraindications but do not provide proper information about the complications and post-treatment precautions.
\end{abstract}

Key words: varicose veins, chronic venous disease, sclerotherapy, complications.

\section{ORIGINAL PAPER}

Phlebological Review 2021; 29, 1: 19-25

D0l: https://doi.org/10.5114/pr.2021.106805

Submitted: 7.05.2021

Accepted: 18.05 .2021

\author{
ADDRESS FOR CORRESPONDENCE \\ Prof. Tomasz Urbanek \\ Department of General Surgery, Vascular \\ Surgery, Angiology, and Phlebology \\ Medical University of Silesia \\ 45/47 Ziołowa St. \\ 40-635 Katowice, Poland \\ e-mail: urbanek.tom@interia.pl
}

\section{INTRODUCTION}

Sclerotherapy is a procedure used in the treatment of telangiectasias, reticular veins, varicose veins, and some other pathologies related to chronic venous disease development and progression [1]. Various techniques of sclerotherapy as well as various agents used for vein obliteration have been proposed [2]. Despite the technical progress and availability of many other minimally invasive methods, sclerotherapy remains one of the most frequently used phlebological treatments and can be based on medical and cosmetic indications.

In comparison with venous surgery, sclerotherapy is often classified as a minimally invasive and relatively safe treatment measure, but, as in the case of every medical procedure, complications are possible as well. Rarely, we have to deal with neurological or visual complications, skin necrosis, deep vein thrombosis, or pulmonary embolism $[1,2]$. Among the most commonly seen complications, skin hyperpigmentation and matting should be mentioned [3]. Proper information about the procedure performance, contraindications, as well as complications is one of the most important steps before the procedure and should be clearly presented to the patients. As in other cases of invasive phlebological treatment, the patient's informed consent should always be obtained. In Poland, similarly to many other countries, due to the lack of the procedure reimbursement, sclerotherapy is often performed in private medical centres and the cost of the procedure is covered by the patient. Looking for treatment options and possibilities, one of the main sources of patient knowledge concerning performance of a particular 
procedure and its possible complications are the websites of centres specializing in sclerotherapy. Unfortunately, the quality of this information remains questionable regarding objective and precise information concerning indications, contraindications and sequalae of the sclerotherapy procedure. Because a lack of patient awareness regarding possible sclerotherapy complications potentially influences the patient's decision, also on acceptance of the sclerotherapy results, the proper quality of information should be provided. The study focuses on investigating how Polish medical centres inform their patients about the potential hazards of sclerotherapy treatment.

\section{MATERIAL AND METHODS}

The website materials of 212 Polish medical centres treating the phlebological patients by the means of sclerotherapy were reviewed and evaluated. The research was carried out by using a specially constructed form in which the data officially provided by medical centres on their websites were compared. The following 5 categories of medical centres were selected: aesthetic clinics (73 facilities $-34.5 \%$ of total), medical centres offering varicose vein treatment (70 facilities - 33\%), medical and aesthetic centres (37 facilities $-17.5 \%$ of total), private specialist practices (20 facilities - 9.5\%), and hospitals (12 facilities $-5.5 \%)$.

The data provided by medical centres were divided into 5 main categories:

- main information about the procedure,

- indication description,

- information about the contraindications,

- post-procedure care,

- possible complications.

The analysed data were collected from clinics' website offers addressed to potential patients interested in sclero-

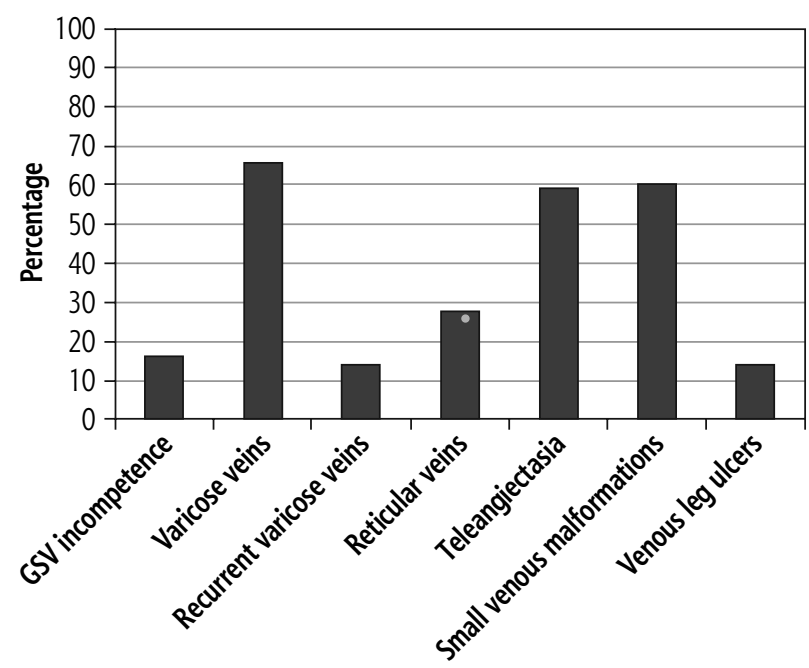

Fig. 1. Indications for sclerotherapy mentioned in the web page information (summarized as the percentage of centres specifying a particular indication) therapy. The main parameter to choose the medical centres was the web page offering sclerotherapy and its high rate in a Google Internet search. This method enabled us to imitate the way in which potential patients search for offers and information about this treatment. We chose the most popular web pages. Medical centres were selected from all voivodeships in Poland. As far as big cities are concerned, the greatest number of centres were found in Warsaw, which is connected to a large population in the capital city.

\section{RESULTS}

\section{General information about sclerotherapy procedure}

Eighty-two per cent of the examined centres explained what sclerotherapy is, and $72 \%$ of them gave details of the procedure. Seventy-seven per cent of the clinics did not inform patients about any special necessary preparations before the treatment, and 53\% did not include any information about the need of performing additional tests such as ultrasonography. As many as $84 \%$ of the centres did not present any information about the possibility of a recovery guarantee, and $58 \%$ of facilities did not inform the patients about possibility of the necessity of re-do procedures or other treatment modalities. This information would be of high potential value for the patient because the lack of a recovery guarantee can prolong the time and increase the costs of treatment.

\section{Indications for sclerotherapy treatment}

Information about indications for sclerotherapy treatment was provided by $74 \%$ of the medical facility websites; however, the least information about indications was placed on private specialist practice web pages - only $45 \%$ of them included any. The most information was presented on the websites of aesthetic and medical clinics. Indications for the treatment were mentioned on $79 \%$ of them. It is worth noting that some medical facilities do not provide any details of indications at all. In the study we focused on the indications shown in Figure 1, and we chose 7 conditions for which sclerotherapy is one of the recommended treatments. The most common indications provided on websites were varicose veins and telangiectasias.

\section{Contraindications}

Overall, only $34 \%$ of medical centres chosen for the evaluation informed the patients and web page visitors about the potential contraindications to the procedure. According to the data presented in Figure 2, in this category the most highly valued are aesthetic clinics, which informed patients about contraindications most frequently, while the information about any contraindications was presented only by $17 \%$ of hospitals. 
The number and kind of the described contraindication differed between the selected groups of centres (Fig. 3) as well as between the individual centres. In this ranking, hospitals compare unfavourably. Among the most commonly mentioned contraindications, the following conditions were identified: pregnancy and lactation, allergy to the drug used for sclerotherapy, as well as deep vein thrombosis. Benign tumours, cancers, and advanced age were more frequently quoted by aesthetic clinics rather than others, and infections with advanced atherosclerosis were provided more often by medical clinics. The type of contraindication as well as the prevalence of this information on the web page of the medical centres is presented in Figure 4.

\section{Post-treatment care}

Information about post-treatment care was present on $68 \%$ of the analysed web sites. The most commonly identified recommendation was the necessity of post-procedure compression stocking application. The majority of facilities, as many as $82 \%$, did not provide any information about avoiding sunbathing and sunscreens, which are among of the most important recommendations after sclerotherapy treatment (Fig. 5).

\section{Procedure complications}

The information about possible sclerotherapy complications could be found on $37 \%$ of analysed websites only. The facilities that informed about the possibility of complications most often were aesthetic medicine centres (40\%), and the least common were the hospital-based centres (Fig. 6).

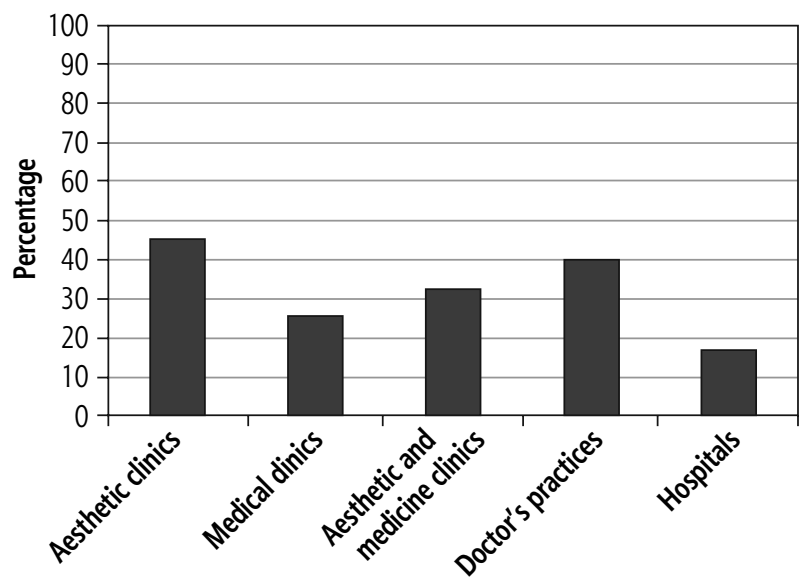

Fig. 2. Facilities providing information about sclerotherapy contraindications (in \%)

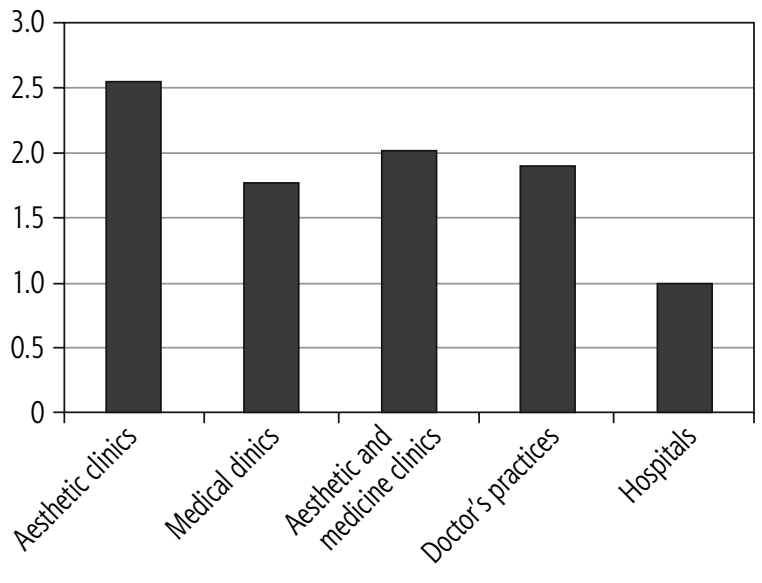

Kind of medical facility

Fig. 3. Average ratio of contraindications provided on a single internet page in the specified centre categories

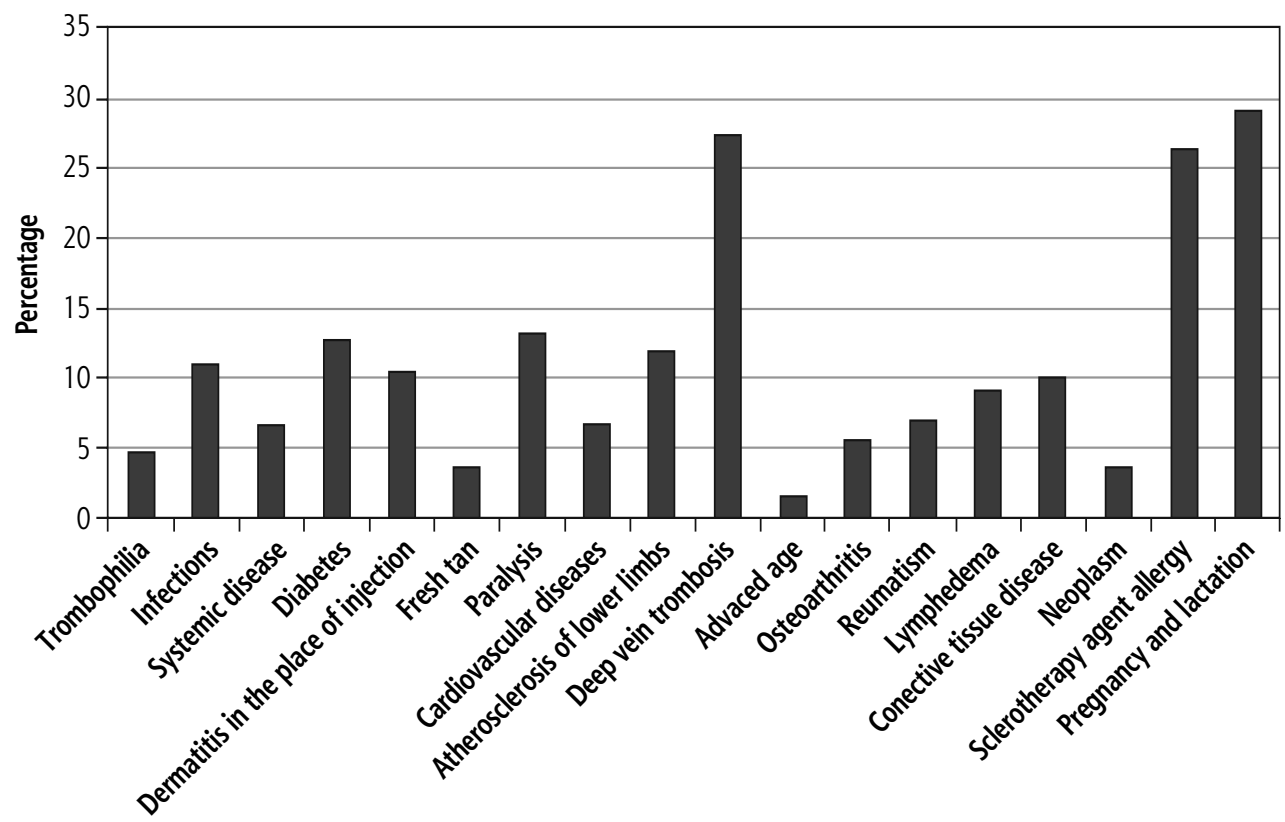

Fig. 4. Contraindications specified in the web page information (\% of the centres evaluated in the study) 


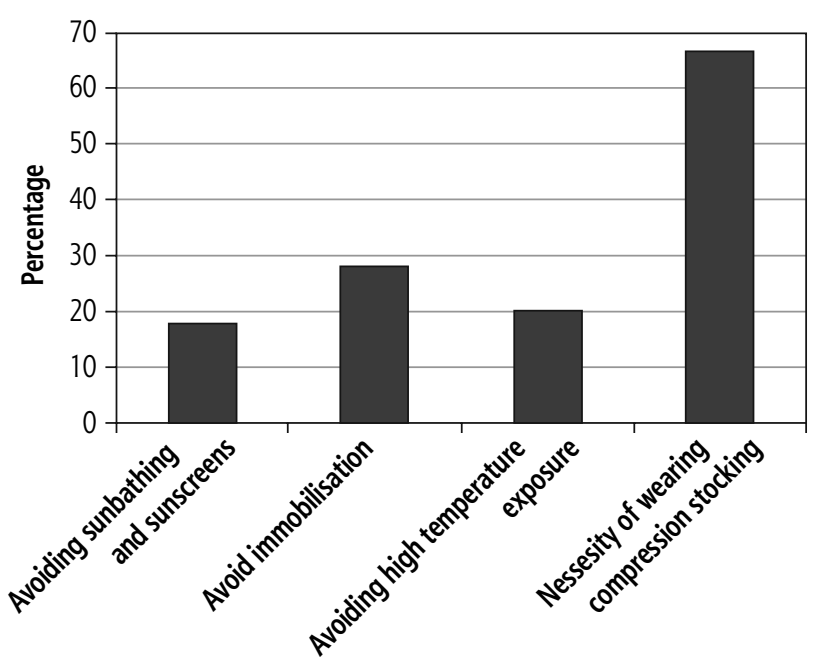

Fig. 5. Post-treatment care information (\% of the centres evaluated in the study)

It is significant that as far as the number of complications mentioned on a single website is concerned, medical clinics compare favourably with aesthetic ones (Fig. 7). On the other hand, as presented in Figure 6, the aesthetic clinics most frequently inform patients about possible complications. The most commonly mentioned complications were haematomas and temporary discoloration of skin, which are among the least harmful sequelae. Information about the dangers of pulmonary embolism, which was presented by only $10 \%$ of analysed facilities and as many as $80 \%$ of medical centres, did not inform patients about possible post-treatment pain. Possible skin loss was scarcely mentioned on the websites; only a few facilities provided information about it (Fig. 8).

\section{DISCUSSION}

Sclerotherapy is a procedure in which varicose veins are obliterated by liquid or foam solutions injected into abnormally expanded or cosmetically unacceptable veins

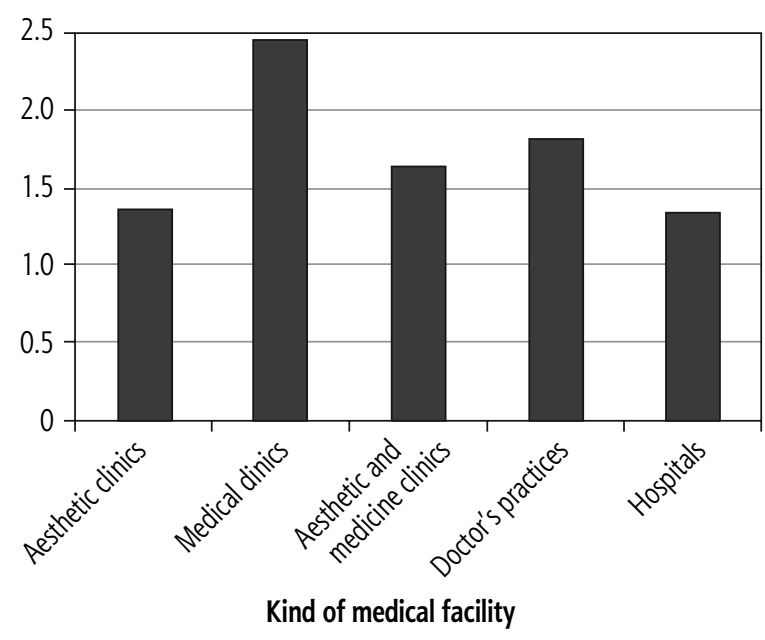

Fig. 7. Number of potential complications per single facility-ratio

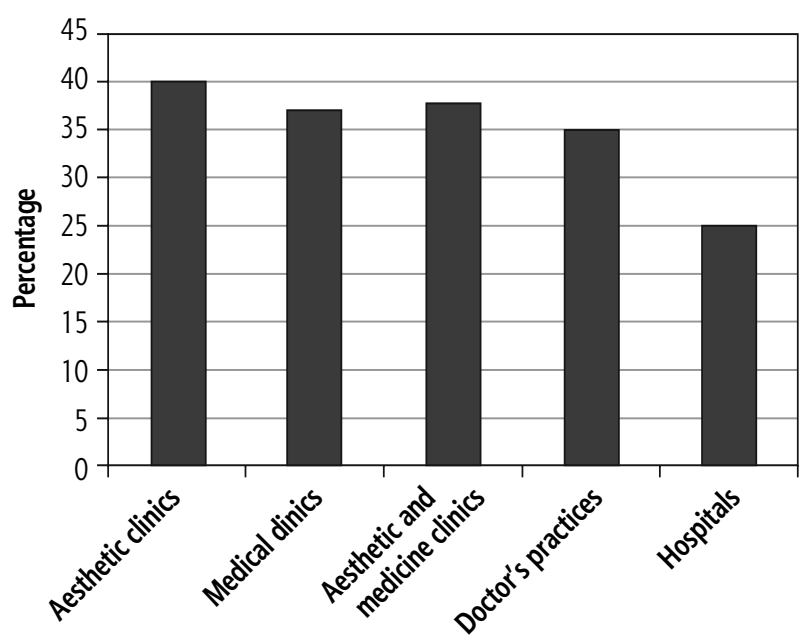

Fig. 6. Percentage of facilities that informed patients about the possibility of complication occurrence

[1-3]. The fact that this procedure has medical and aesthetic advantages is beyond any doubt [4]. Because the sclerotherapy costs are not covered by the national insurance system in Poland, most of the sclerotherapy procedures are performed in private centres and hospitals and are fully paid for by the treated patients. The introduction of foam sclerotherapy as well as ultrasound-guided sclerotherapy significantly expanded the possibilities of minimally invasive treatment of venous disease by the means of this therapeutic measure. The efficacy of sclerotherapy in superficial vein incompetence treatment has been confirmed in several prospective observations [5-12].

The minimal invasive procedure character, the possibility to treat a wide spectrum of venous pathologies, as well as the relatively low cost of the treatment, position sclerotherapy among the most commonly used therapeutic tools in the contemporary phlebology. Despite the growing experience as well as the growing number of the sclerotherapy procedures worldwide, sclerotherapy is not always completely successful, and at least in some of the patients treatment failure can be expected $[5,8,10]$. The lack of clinical success of the sclerotherapy procedure can be related to improper patient qualification, improper procedure performance, and to vein recanalization or venous disease progression [13-16]. In the Rasmussen et al. study, 1 year after ultrasound-guided foam sclerotherapy, $16.3 \%$ of the saphenous veins remained patent and refluxing [15]. Shadid documented saphenous vein reopening 2 years after ultrasound-guided sclerotherapy in $11.3 \%$ of cases. The fact that sclerotherapy is not always the final treatment, and in future we can expect residual varicose veins or disease recurrence, is worth mentioning because in some cases the need for repeated treatment occurs. Fifty-eight per cent of the centres evaluated in our study did not inform patients on their web pages about the potential necessity of re-do procedures or other treatment modalities. Despite the fact that this situation can 


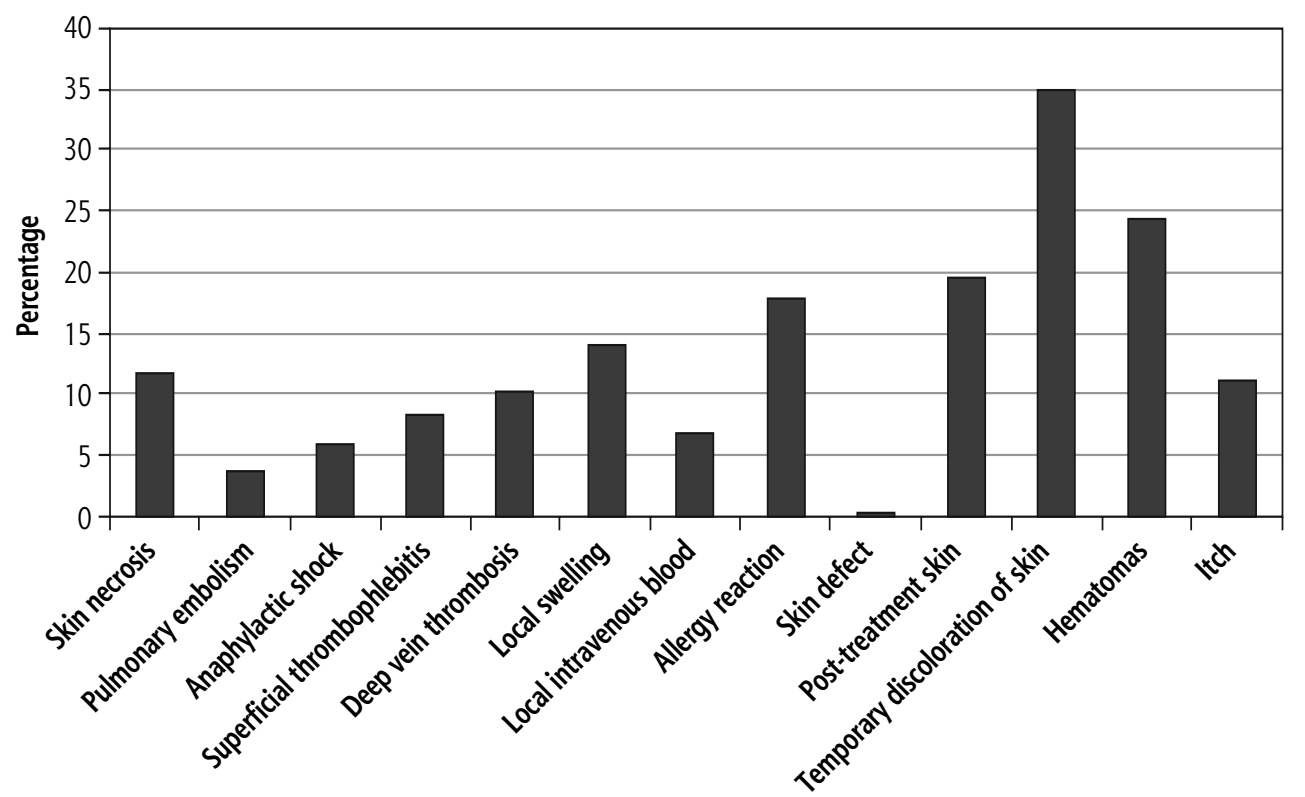

Fig. 8. Complications specified in the web page information (\% of the centres evaluated in the study)

prolong the treatment duration and potentially increase both the treatment costs and the need for the additional procedure performance, this information is commonly skipped in the available webpage materials.

Information about medical indications is a very important part of every offer, and it allows the patient to decide about the need for this treatment $[3,17,18]$. According to the European Sclerotherapy Guidelines (2012), several indications for sclerotherapy performance can be mentioned, including saphenous vein incompetence (grade $1 \mathrm{~A}$ recommendation), tributary incompetence (1B), residual or recurrent varicose veins (1B), reticular veins and or telangiectasias (1A), perforating veins $(1 \mathrm{~B})$, incompetent veins in venous leg ulcer patients (1B), as well as venous malformations (1B) [3]. In the performed study, information about the potential indication for the sclerotherapy performance was present, on average, on $74 \%$ of the medical facility websites. However, more than half of the available patient materials presented on private specialist practice web pages did not include any indications. The most common indications provided on the evaluated websites were varicose veins and telangiectasias, with significant differences between the medical and aesthetic centres regarding the number of the indications.

Only $34 \%$ of the medical centres chosen for the evaluation informed the web page visitors about the possible contraindications to the procedure. The results show that most information about contraindications come from facilities belonging to the category of aesthetic clinics, but information about them is very rarely provided by hospitals. Unfortunately, a small percentage of facilities mentioned individual contraindications, although here also aesthetic clinics are better than others. Among the most commonly mentioned, pregnancy, lactation, deep vein thrombosis, and allergy to the sclerosing agent were specified. In the vast majority of the centres, other important contraindications such as acute infection, lack of the possibility of medical compression application, as well as patent foramen ovale with right to left shunt were not mentioned $[3,18]$. Information about the recommended post-procedure action was provided by more than half of the analysed facilities. The majority of medical centres recommended wearing medical compression tight length stocking. We rarely found information about avoiding high temperatures and avoiding immobilization, and the even less often, information about avoiding self-tanning creams and sunbathing. These actions require self-discipline and very often make patients completely change their habits, which can affect their decision about undergoing sclerotherapy treatment.

Sclerotherapy, like every procedure, includes the risk of many side effects $[8,9]$. Because sclerotherapy is divided into 2 major kinds - liquid and foam - there are different risks of side effects $[3,18,19-22]$. Foam sclerotherapy has a relatively high percentage of complications, but it can achieve higher stability of clinical effectiveness in treating varicose veins of lower limbs than liquid sclerotherapy [3, 11, 19, 20]. Several sclerotherapy adverse effects have been reported, and the procedure complications can be divided into common, uncommon, or rare (Table 1, 2).

Unfortunately, only a few centres (37\%) in our study discussed the subject of the complications on their web pages. The research results show us a divergence, because in this category the best were aesthetic clinics. However, if we analyse the number of side effects provided on a single web page, the medical clinics were the most informative. 
Table 1. Possibility of side effects after sclerotherapy [2, 3]

\begin{tabular}{lc}
\hline Designation & Incidence \\
\hline Very common & $\geqslant 10 \%$ \\
\hline Common & $\geqslant 1 \%-<10 \%$ \\
\hline Uncommon & $\geqslant 0.1 \%-<1 \%$ \\
\hline Rare & $\geqslant 0.01 \%-<0.1 \%$ \\
\hline Very rare and isolated case & $<0.01 \%$ \\
\hline
\end{tabular}

Table 2. Possibility of side effects after sclerotherapy

\begin{tabular}{lcc}
\hline Type of complication & $\begin{array}{c}\text { Frequency with } \\
\text { liquid }\end{array}$ & $\begin{array}{c}\text { Frequency with } \\
\text { foam }\end{array}$ \\
\hline \begin{tabular}{l} 
Severe complications \\
\hline Anaphylaxis
\end{tabular} & Isolated cases & Isolated cases \\
\hline Large tissue necrosis & Isolated cases & Isolated cases \\
\hline Proximal deep vein thrombosis & Very rare & Very rare \\
\hline Pulmonary embolism & Isolated cases & Isolated cases \\
\hline Benign complications & & \\
\hline Headaches and migraines & Very rare & Uncommon \\
\hline Skin reaction (local allergy) & Very rare & Very rare \\
\hline Matting & Common & Common \\
\hline Residual pigmentation & Common & Common \\
\hline Skin necrosis & Rare & Very rare \\
\hline
\end{tabular}

We can assume that evaluated aesthetic clinics did not provide very specific information about side effects but they only focused on the fact that they exist. Considering the frequency of complications, we can notice that offers provide only information about the most common and least dangerous ones and occasionally mention rare and very dangerous side effects such as pulmonary embolism, which can be lethal. Some limitations of our study should also be mentioned. The study was based on the webpage information available for potential sclerotherapy customers, but it did not include the content of the information for the patient or the information contained in the patient informed consent form signed by the patients before the procedure. Because we did not have access to these documents from the individual centres, we approached their web materials as patients in the initial phase of their decision making do. Of course, we can expect that the information provided during the medical qualification visit is more precise and differs from the web page information. Another important limitation of our research is the fact that many of the sclerotherapy specialists in our country still do not have a dedicated web page that could be a source of information for potential patients, and these centres were not available for the potential information analysis.

\section{CONCLUSIONS}

To attract patients, several medical centres inform them only about the indications rather than contraindications, and they do not provide proper information about the complications and post-treatment precautions. The way in which information about every aspect of sclerotherapy is provided constitutes evidence that this intervention is disregarded, treated rather like a beauty treatment and available for everyone. Unfortunately, this intervention is usually covered financially by patients, which is strongly connected with the interest of profit-driven clinics offering sclerotherapy. Most of their offers look like advertisements or commercial spots, and only few of them focus on crucial medical facts.

\section{The authors declare no conflict of interest.}

\section{References}

1. Weiss MA, Hsu JT, Neuhaus I, Sadick NS, Duffy DM. Consensus for sclerotherapy. Dermatol Surg. 2014; 40: 1309-1318.

2. Guex JJ. Complications of sclerotherapy: an update. Dermatol Surg. 2010; 36: 1056-1063.

3. Rabe E, Breu F, Cavezzi A, et al. European guidelines for sclerotherapy in chronic venous disorders. Phlebology 2014; 29: 338-354.

4. Khunger N, Sacchidanand S. Standard guidelines for care: sclerotherapy in dermatology. Indian J Dermatol Venereol Leprol 2011; 77: 222-231.

5. Rabe E, Schliephake D, Otto J, Breu FX, Pannier F. Sclerotherapy of telangiectasias and reticular veins: a double-blind, randomized, comparative clinical trial of polidocanol, sodium tetradecyl sulphate and isotonic saline (EASI study). Phlebology 2010; 25: 124-131.

6. Kahle B, Leng K. Efficacy of sclerotherapy in varicose veins a prospective, blinded placebo controlled study. Dermatol Surg 2004; 30: 723-728.

7. Ouvry P, Allaert FA, Desnos P, Hamel-Desnos C. Efficacy of polidocanol foam versus liquid in sclerotherapy of the great saphenous vein: a multicenter randomised controlled trial with a 2-year follow-up. Eur J Vasc Surg 2008; 36: 366-370.

8. Kakkos SK, Bountouroglou DG, Azzam M, Kalodiki E, Daskalopoulos M, Geroulakos G. Effectiveness and safety of ultrasound-guided foam sclerotherapy for recurrent varicose veins: immediate results. J Endovasc Ther 2006; 13: 357-364.

9. Coleridge Smith P. Chronic venous disease treated by ultrasound guided foam sclerotherapy. Eur J Vasc Endovasc Surg 2006; 32: 577-583.

10. Paraskevas P. Successful ultrasound-guided foam sclerotherapy for vulval and leg varicosities secondary to ovarian vein reflux: a case study. Phlebology 2011; 26: 29-33.

11. Cavezzi A, Frullini A, Ricci S, Tessari L. Treatment of varicose veins by foam sclerotherapy: two clinical series. Phlebology 2002; 17: 13-18.

12. Pang KH, Bate GR, Darvall KAL, Adam DJ, Bradbury AW. Healing and recurrence rates following ultrasound guided foam sclerotherapy of superficial venous reflux in patients with chronic venous ulceration. Eur J Vasc Endovasc Surg 2010; 40: 790-795. 
13. Chen $\mathrm{CH}$, Chiu CS, Yang CH. Ultrasound-guided foam sclerotherapy for treating incompetent great saphenous veins - results of 5 years of analysis and morphologic evolvement study. Dermatol Surg 2012; 38: 851-857.

14. Rabe E, Otto J, Schliephake D, Pannier F. Efficacy and safety of great saphenous vein sclerotherapy using standardised polidocanol foam (ESAF): a randomised controlled multicentre clinical trial. Eur J Endovasc Vasc Surg 2008; 35: 238-245.

15. Rasmussen LH, Lawaetz M, Bjoern L, Vennits B, Blemings A, Eklof B. Randomized clinical trial comparing endovenous laser ablation,radiofrequency ablation, foam sclerotherapy and surgical stripping for great saphenous varicose veins. Br J Surg 2011; 98: 1079-1087.

16. Shadid N, Ceulen R, Nelemans P, et al. Randomized clinical trial of ultrasound-guided foam sclerotherapy versus surgery for the incompetent great saphenous vein. Br J Surg 2012; 99: 1062-1070.

17. Rabe E, Partsch H, Hafner J, et al. Indications for medical compression stockings in venous and lymphatic disorders: an evidence-based consensus statement. Phlebology 2018; 33: 163-184.

18. Rabe E, Pannier F. Sclerotherapy of varicose veins with polidocanol based on the guidelines of the German Society of Phlebology. Dermatol Surg 2010; 36: 968-975.

19. Gibson K, Gunderson K. Liquid and foam sclerotherapy for spider and varicose veins. Surg Clin North Am 2018; 98: 415-429.

20. Bi M, Li D, Chen Z, et al. Foam sclerotherapy compared with liquid sclerotherapy for the treatment of lower extremity varicose veins: a protocol for systematic review and meta-analysis. Medicine (Baltimore) 2020; 99: e203327.

21. Yiannakopoulou E. Safety concerns for sclerotherapy of telangiectases, reticular and varicose veins. Pharmacology 2016; 98 : 62-69.

22. Subbarao NT, Aradhya SS, Veerabhadrappa NH. Sclerotherapy in the management of varicose veins and its dermatological complications. Ind J Dermatol Venereol Leprol 2013; 79: 383-388. 